\title{
3D Printed Microscope Optomechanical Tools
}

Jeffrey Kuhn

Koch Institute for Integrative Cancer Research at MIT, Cambridge, Massachusetts, United States

Discovery often involves the creation of new apparatuses by curious minds and industrious hands. From Leeuwenhoek's simple microscopes to a modern particle collider, science has always been a 'maker' movement. Biology is no exception, especially in the realm of new instruments for microscopy.

Basic cell discoveries or new cancer therapies require novel, faster, and cheaper light microscopy methods. Development of these new methods almost invariably requires custom optomechanics, lens holders, and adapters. Even with automated computer numerical control (CNC) machining, iteration time between part design, metal machining, and testing can take weeks to months per cycle with accompanying high labor costs and large capital investments in equipment.

The wide availability of inexpensive 3D printers for filament deposition modeling (FDM) have dramatically shortened iteration times in microscope part design. Optomechanical designs that incorporate printed parts can be rapidly and repeatedly updated as the design evolves.

There has been an open-source explosion in 3D printed optics and microscopes. Printed parts offer excellent supplements to microscope frames [1]-[3] and traditional cage, rail, and post systems [4]-[7]. FDM printed parts can help researchers rapidly test spatial limitations and adapt instruments with optical paths.

Despite their benefits, 3D printed parts are not direct substitutes for traditional optomechanics. Plastic thermal expansion, deposition tolerances, low tensile strengths, and the layer-by-layer process itself place design constraints on FDM printed parts. I will discuss design and printing strategies for incorporating FDM printed parts into both existing and new optomechanical systems.

- Always design parts with tolerances in mind. The extrusion process is inexact and plastic shrinks as it cools. Parts printed to fit together or with existing machined parts must be designed and tested for fit tolerances rather than for exact mechanical dimensions.

- Design optomechanical parts that self-center using radially symmetric tolerance where axial alignment is critical. For example, optical rail slots can be lengthened slightly perpendicular to the optical axis to offer room for part shrinkage (Fig 1).

- Bottom-up layer-by-layer filament deposition requires support structure underneath spans of more than a few millimeters. Design parts with gradual overhangs or with removable support structures where possible.

- Metal machine screws with printed female threads in parts are adequate for one-time assembly or occasional adjustment. Machine nut catches should be use for frequent reassembly.

- Only the bottom surface of printed parts will be flat. The cross-hatch pattern generated by most slicing algorithms produces surface roughness that do not work as friction bearings. Instead, grooves can be designed into top surfaces to force slicing software to generate concentric or parallel layers adequate for sliding or rotating friction bearings (Fig 2). 


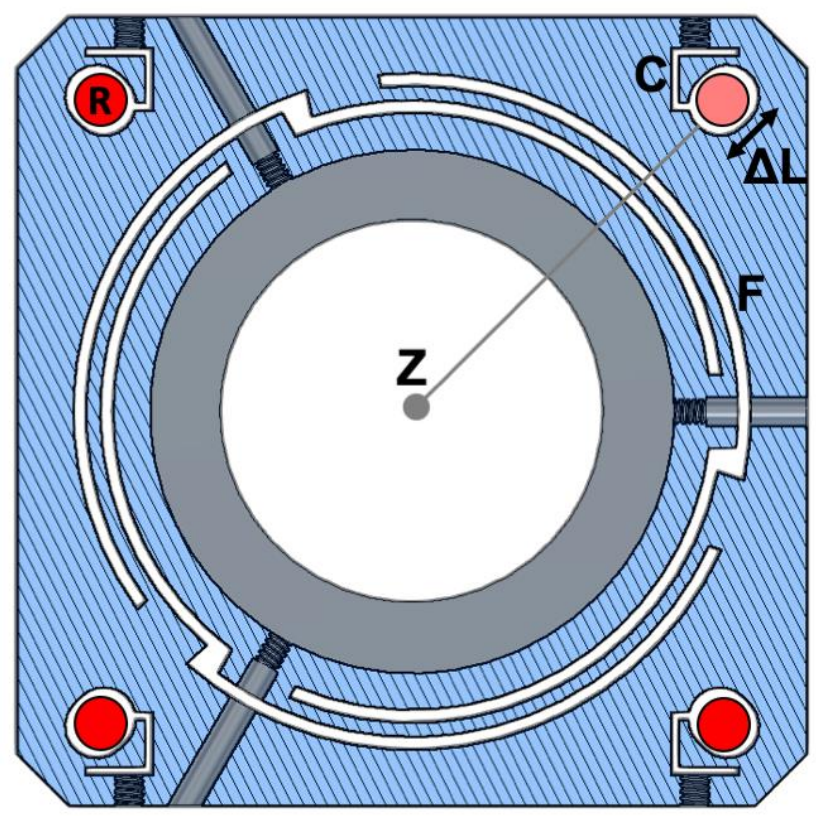

Figure 1. Printed self-centering optical plate. Cross section view of optical shutter mount for an optical cage system containing four rods $(\mathrm{R})$. Hole tolerance $\Delta \mathrm{L}$ is radially extended to compensate for plastic part shrinkage. Because all four rod holes shrink toward the optical axis $\mathrm{Z}$, dimensional adjustment between machined cage plates and printed cage plates is maintained after printing. A shutter is mounted at the center using three set screws and flexures (F) for vibration dampening. Plastic flexures and setscrews are also used for rod clamps (C).

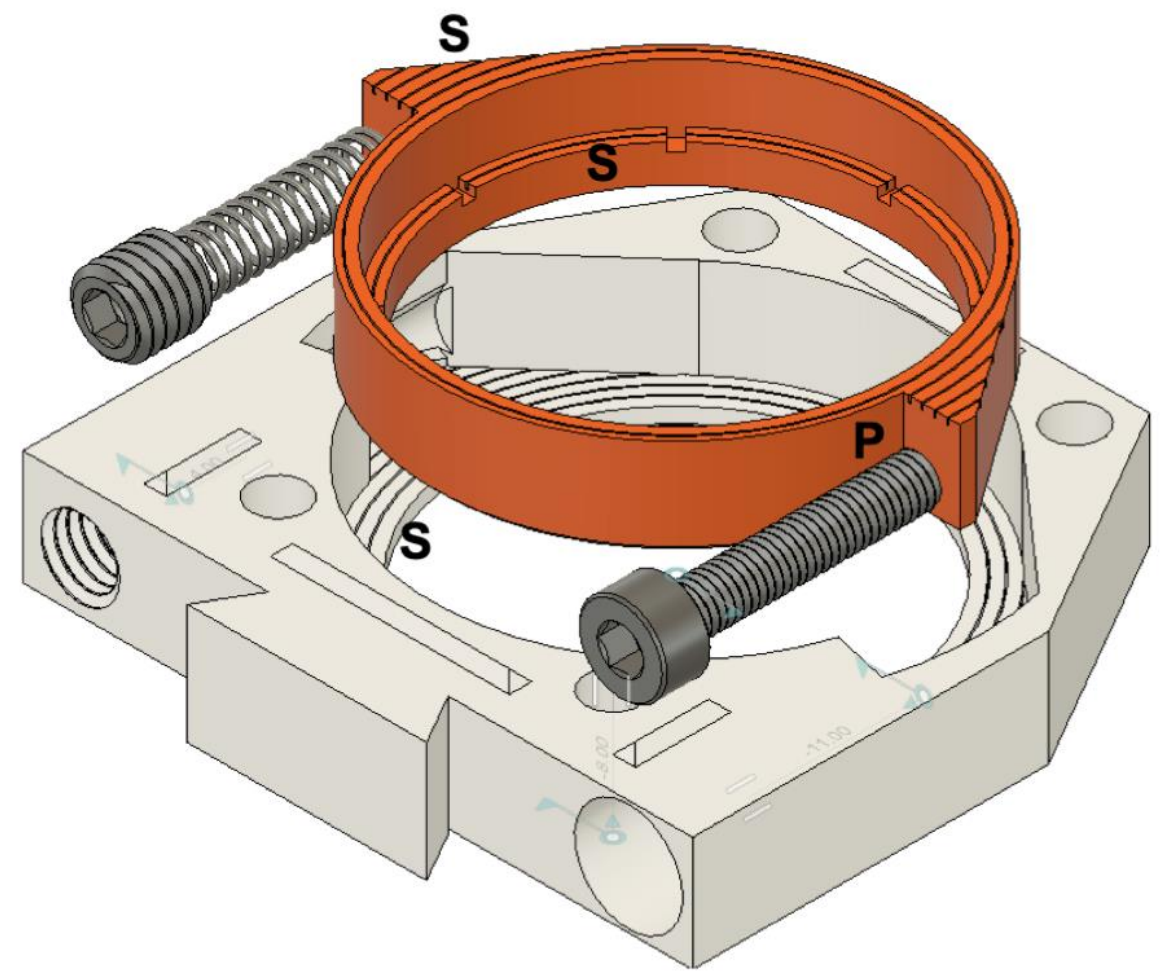

Figure 2. Adjustable, stackable waveplate mounts. Smooth friction bearings (S) can be engineered into the top surfaces of parts by designing groves that force slicing software to print the top in concentric rings. 
An opposing screw and spring mechanism allowed an internal waveplate pusher (P) to rotate by a few degrees for matching. Three of these 1-inch waveplate mounts stack into a unit the size of a standard Nikon fluorescent filter cube.

\section{References}

[1] B. Diederich et al., "UC2 - A 3D-printed General-Purpose Optical Toolbox for Microscopic Imaging," p. ITh3B.5, 2019, doi: 10.1364/isa.2019.ith3b.5 .

[2] J. T. Collins et al., "Robotic microscopy for everyone: the OpenFlexure Microscope," Biorxiv, p. 861856, 2019, doi: 10.1101/861856 .

[3] O. University and L. Hughes, "3D printing in microscopy and science outreach," Infocus Mag, pp. 1828, 2015, doi: 10.22443/rms.inf.1.128 .

[4] A. Lambert, S. Valiulis, and Q. Cheng, “Advances in Optical Sensing and Bioanalysis Enabled by 3D Printing," Acs Sensors, vol. 3, no. 12, pp. 2475-2491, 2018, doi: 10.1021/acssensors.8b01085 .

[5] B. J. Winters and D. Shepler, “3D printable optomechanical cage system with enclosure," Hardwarex, vol. 3, no. Open-Source Hardware. Science 3376100 2012, pp. 62-81, 2017, doi: 10.1016/j.ohx.2017.12.001 .

[6] L. Salazar-Serrano, J. P. Torres, and A. Valencia, "A 3D Printed Toolbox for Opto-Mechanical Components," Plos One, vol. 12, no. 1, p. e0169832, 2017, doi: 10.1371/journal.pone.0169832 .

[7] C. Zhang, N. C. Anzalone, R. P. Faria, and J. M. Pearce, "Open-Source 3D-Printable Optics Equipment," Plos One, vol. 8, no. 3, p. e59840, 2013, doi: 10.1371/journal.pone.0059840 . 